\title{
Ambipolar silicon nanowire FETs with stenciled-deposited metal gate
}

\author{
Davide Sacchetto*, Veronica Savu, Giovanni De Micheli, Jürgen Brugger, Yusuf Leblebici \\ EPFL-Ecole Polytechnique Fédérale de Lausanne, 1015, Vaud, Switzerland
}

\section{A R T I C L E I N F O}

\section{Article history:}

Available online 5 January 2011

\section{Keywords:}

Schottky barrier

Ambipolarity

Si nanowire

Stencil lithography

FET

Silicide

\begin{abstract}
A B S T R A C T
We report on a fully CMOS compatible fabrication method for ambipolar silicon nanowire FinFETs. The low thermal budget processing, compatible with monolithic 3D device integration, makes use of low pressure chemical vapor deposition (LPCVD) of amorphous $\mathrm{Si}\left(\mathrm{a}-\mathrm{Si}\right.$ ) and $\mathrm{SiO}_{2}$ layers as well as metal gate patterning using stencil lithography, demonstrated for the first time. FinFETs with stenciled Al gates are successfully co-fabricated with polycrystalline silicon $\Omega$-gated devices. Stencil lithography is envisaged as a key enabler for gate patterning on 3D structures, such as vertically stacked nanowire transistors.
\end{abstract}

(c) 2011 Elsevier B.V. All rights reserved.

\section{Introduction}

Future technological innovations enabling ever higher circuit densities predicted by Moore's law will most likely be concentrated on novel materials, innovative device structures or different state variables other than charge [1]. All these approaches will lead to significant modifications of the traditional planar transistor design. Considering novel device structures, the nanowire (NW) channel transistor with FinFET construction has been demonstrated to be one of the best in terms of electrostatic control, thus enabling further device scaling [2]. Another example can be the monolithic 3D integration, a process integration scheme that achieves higher device density by stacking p-type FETs on top of n-type FETs [3]. In terms of state variables, one example is given by device ambipolarity. The ambipolar property can be described by the presence of two high conductance states for the same FET, each of these related to carriers of opposite charge values [4]. Preliminary works on device ambipolarity show how it can be used to compute more complex logic functions per chip [5]. However, in terms of process integration, a few technologies have the flexibility to be implemented within 3D integration schemes and, at the same time, offer the ambipolar property with good CMOS compatibility and electrical performance.

We report on a fully CMOS compatible fabrication of ambipolar SiNW FinFETs by means of amorphous $\mathrm{Si}$ (a-Si) and low temperature oxide (LTO) deposited with low pressure chemical vapor deposition (LPCVD) technique. FinFETs with stenciled Al gates are successfully co-fabricated with polycrystalline $\mathrm{Si}$ (pc-Si) gated devices achieving excellent performance. The difficulty of

\footnotetext{
* Corresponding author.

E-mail address: davide.sacchetto@epfl.ch (D. Sacchetto).
}

achieving high resolution gate patterning on non-planar devices is addressed for the first time by means of stencil metal deposition [6]. We envisage the use of this technique also for metal patterning on high aspect ratio FETs, such as vertically stacked nanowire transistors [7]. It is worth noting that this process can be repeated several times, eventually being used for monolithic 3D integration.

In Section 2 we present the fabrication steps to build the devices. Then in Section 3 the fabricated structures are shown and described. In Section 4 we describe the electrical behavior of the two types of FinFETs fabricated and on the pseudo-inverter operation of pc-Si FinFETs. Finally, in Section 5 we draw the conclusions.

\section{Process flow}

The fabrication process starts with the formation of isolation and active layers on a bulk-Si substrate. The isolation is composed of a $500 \mathrm{~nm}$ thick wet oxide and a $100 \mathrm{~nm}$ LTO layer. Then a $100 \mathrm{~nm}$ a-Si film is deposited as the active layer. A $70 \mathrm{~nm}$ thick dilution of hydrogen silsesquioxane (HSQ) is spin coated and patterned with e-beam lithography. Lines having widths ranging from $70 \mathrm{~nm}$ to $250 \mathrm{~nm}$ have been used as mask for Si dry etching. An LTO under etch and HSQ strip has been performed by a dip buffered hydrofluoridric acid (BHF) step. The obtained a-Si nanowires are then covered by a $40 \mathrm{~nm}$ dry oxide and a $50 \mathrm{~nm}$ LPCVD pc-Si layers. Large $100 \mu \mathrm{m} \times 100 \mu \mathrm{m}$ pc-Si pads and gates are etched with an anisotropic $\mathrm{SF}_{6}$ plasma etching recipe. Thus, pc-Si $\Omega$-gated FinFETs with gate lengths between $1 \mu \mathrm{m}$ and $10 \mu \mathrm{m}$ are obtained.

Then a bi-layer of $20 \mathrm{~nm} \mathrm{Ti} / 55 \mathrm{~nm} \mathrm{Ni}$ is patterned with lift-off to form electrical contacts between the Si nanowires and the pads. A $400{ }^{\circ} \mathrm{C}$ furnace annealing step forms $\mathrm{Ni}_{x} \mathrm{Si}_{y}$ silicide source and drain Schottky junctions at the metal/Si interface. The Ti layer serves as cap layer to prevent $\mathrm{Ni}$ oxidation. $\mathrm{Ni}$ silicide process has been 
chosen for its mid-gap work function and for its use in a-Si metal induced recrystallization process [8]. The fabrication flow is completed by the patterning of Al-gated devices by means of a stencil deposition approach. The full wafer stencil mask contains $100 \mathrm{~nm}$ thick SiN membranes with apertures having widths between

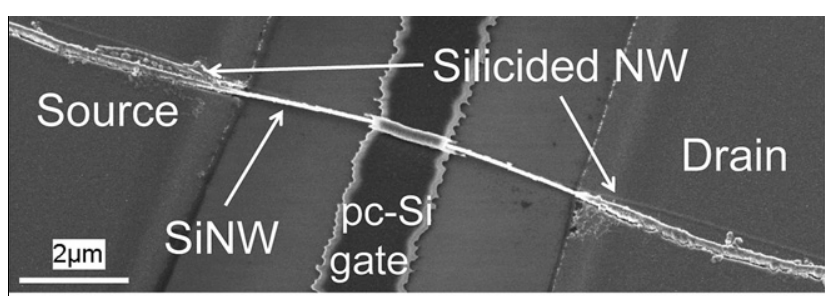

(a)

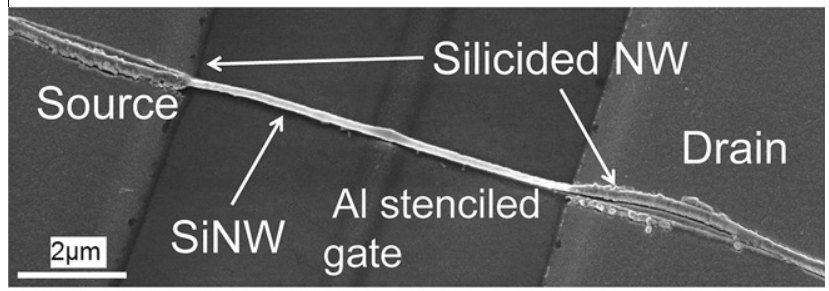

(b)

Fig. 1. FinfETs with silicide nanowire portions at source/drain contacts: (a) pc-Si FinFET having $2 \mu \mathrm{m}$ long gate and $60 \mathrm{~nm} \times 65 \mathrm{~nm}$ channel cross-section. (b) Al stenciled FinFET having $700 \mathrm{~nm}$ gate length and $60 \mathrm{~nm} \times 140 \mathrm{~nm}$ channel crosssection.
$100 \mathrm{~nm}$ and $1 \mu \mathrm{m}$ [9]. Through these, material for the transistor gates is deposited. The stencil is manually aligned to the substrate with $2 \mu \mathrm{m}$ [9] accuracy using a customized SUSS MA/BA 6 mask aligner [10]. The clamped substrate-stencil setup is placed in an evaporator where $100 \mathrm{~nm}$ thick $\mathrm{Al}$ gates are deposited.

\section{Fabricated structures}

FinFETs and inverters with up to $62 \mu \mathrm{m}$ nanowire length were fabricated. An example of a $2 \mu \mathrm{m}$ long pc-Si gate FinFET having $100 \mathrm{~nm} \times 65 \mathrm{~nm}$ Si nanowire channel is shown in the SEM tilted view of Fig. 1(a). In Fig. 1(b), a Si nanowire transistor with $130 \mathrm{~nm} \times 65 \mathrm{~nm}$ channel (see Fig. 2(d)) cross-section and $700 \mathrm{~nm}$ large stenciled $\mathrm{Al}$ gate is shown. Focused ion beam (FIB) cross-sections (see Fig. 2(a)-(c)) demonstrate nanowire channels sections having widths between $65 \mathrm{~nm}$ and $130 \mathrm{~nm}$. The total Si thickness is reduced from the initial value of $100 \mathrm{~nm}$ to $65 \mathrm{~nm}$ due to processing. Narrower nanowires (the expected nanowire size was around $35 \mathrm{~nm}$ ) detached from the isolation layer due to LTO under etch caused by the dip BHF step.

\section{Electrical measurements and discussion}

The $I_{\mathrm{ds}}-V_{\mathrm{gs}}$ curve of a pc-Si $\Omega$-gate FinFET has a typical ambipolar behavior, with a higher conductance for holes (at negative $V_{\mathrm{gs}}$ voltages) than for electrons (Fig. 3(a)). In the holes-conductance regime, a subthreshold slope $\mathrm{SS} \approx 420 \mathrm{mV} / \mathrm{dec}$ is observed, while a larger SS is observed for the electron-dominated current. The gate misalignment error is source of variation for the channel series

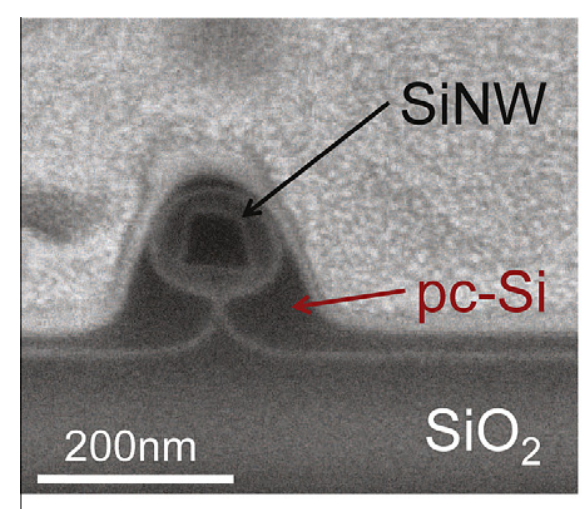

(a)

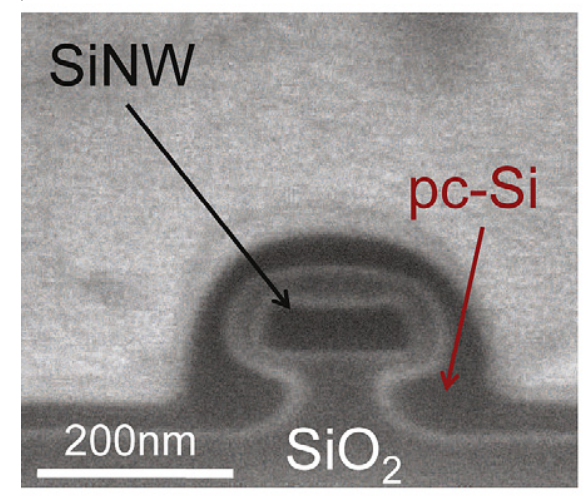

(c)

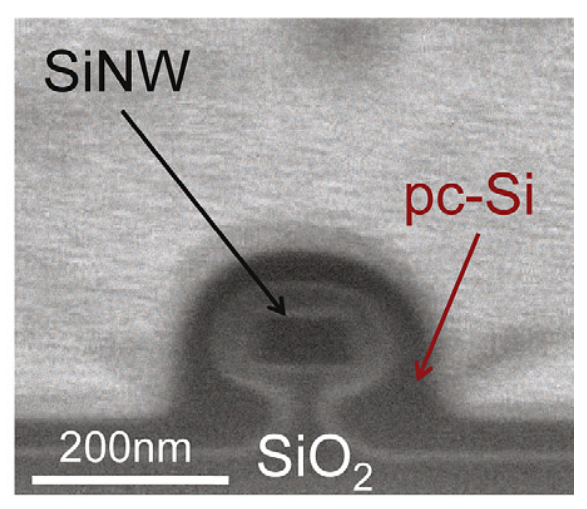

(b)

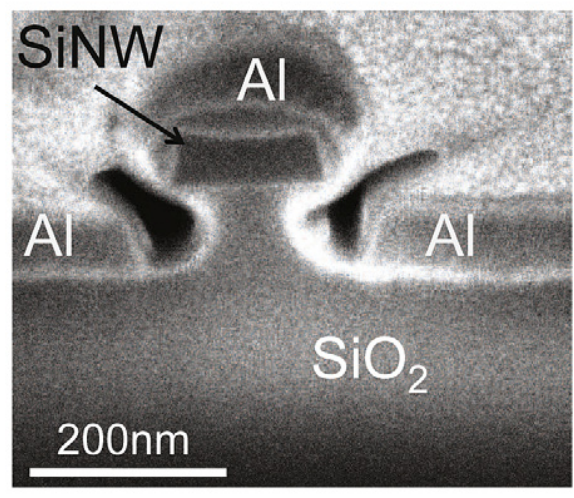

(d)

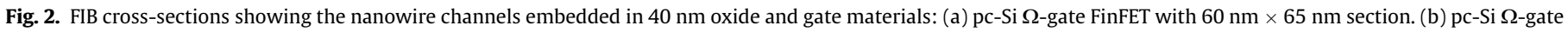
FinFET with $60 \mathrm{~nm} \times 110 \mathrm{~nm}$ section. (c) pc-Si $\Omega$-gate FinFET with $60 \mathrm{~nm} \times 140 \mathrm{~nm}$ section. (d) Al stenciled-gate FinFET with $60 \mathrm{~nm} \times 140 \mathrm{~nm}$ section. 


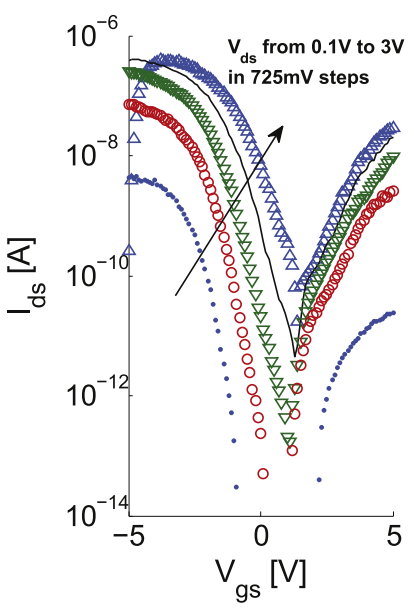

(a)

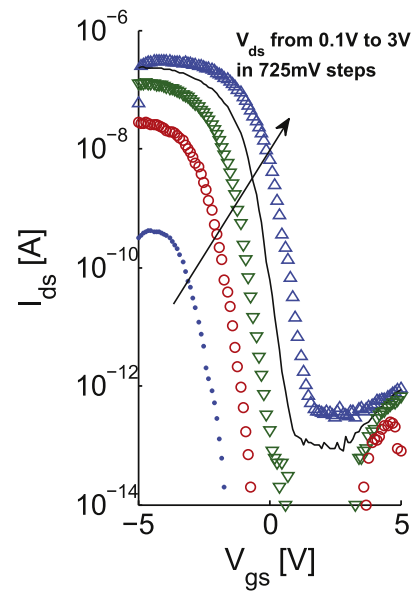

(b)
Fig. 3. (a) Ambipolar $I_{\mathrm{d}}-V_{\mathrm{g}}$ curve of a pc-Si $\Omega$-gated FinFET having $1 \mu \mathrm{m}$ gate length and $60 \mathrm{~nm} \times 110 \mathrm{~nm}$ nanowire section. (b) Ambipolar $I_{\mathrm{d}}-V_{\mathrm{g}}$ curve of a stenciled Al-gate FinFET having $1 \mu \mathrm{m}$ gate length and $60 \mathrm{~nm} \times 110 \mathrm{~nm}$ nanowire section.

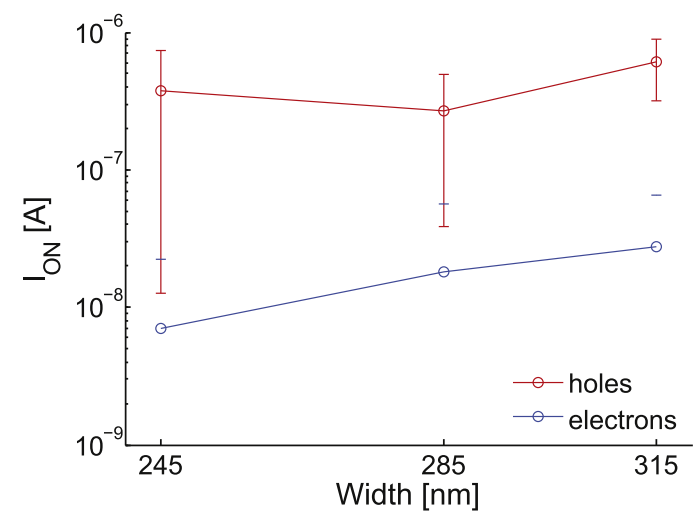

Fig. 4. Average $I_{\mathrm{ON}}$ for holes and electrons of pc-Si $\Omega$-gated FinFETs of different widths. The error bar represents the standard deviation over a sample of eight devices.

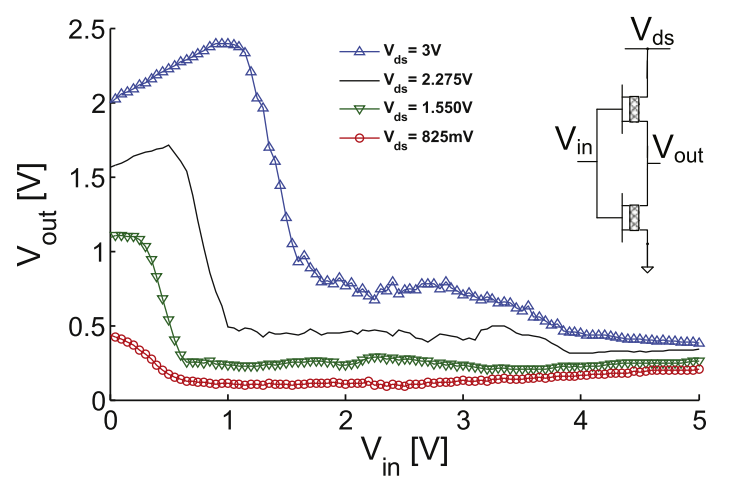

Fig. 5. Voltage transfer characteristic of a 2 in series connected pc-Si $\Omega$-gate FinFETs on-a-wire and biased as for inverter operation. The insets represent the inverter bias scheme. Two ambipolar pc-Si FinFETs are connected in series.

resistance which in turn impacts the overall current conductance. Nevertheless, the main limiting factor for the current conductance is the injection of carriers over the Schottky barrier, and the series resistance is considered to play a minor role. This aspect is

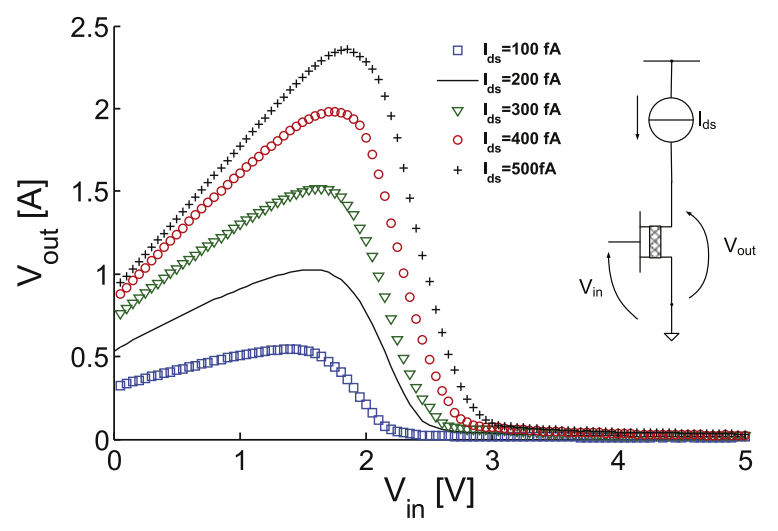

Fig. 6. pc-Si FinFET biased for pseudo-inverter operation having $2 \mu \mathrm{m}$ gate lengths and $60 \mathrm{~nm} \times 110 \mathrm{~nm}$ channel cross-section. The insets show the pseudo-inverter bias scheme using a single pc-Si FinFET and a current source.

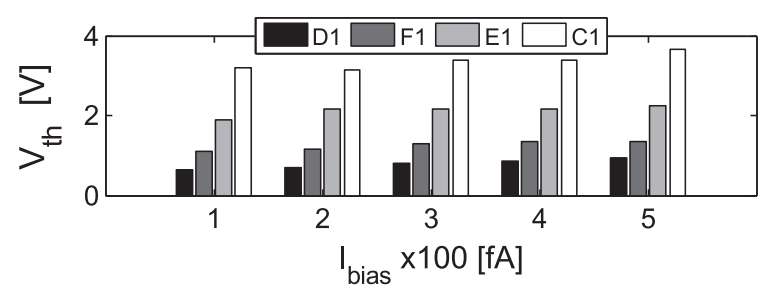

(a)

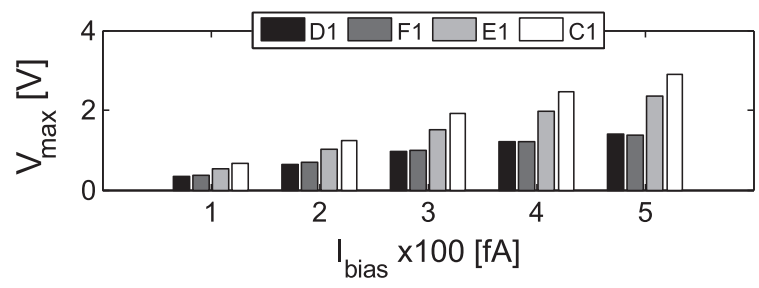

(b)

Fig. 7. (a) Threshold voltage $\left(V_{\text {th }}\right)$ values for the pc-Si gated pseudo-inverters. The inversion voltages are stable under different current bias. (b) Maximum output voltages values for the pc-Si gated pseudo-inverters. The $V_{\max }$ values tend to increase linearly with $I_{\text {bias }}$.

confirmed by the observation of a lower $I_{\mathrm{ON}}$ for electrons than holes over a large number of samples. In Fig. 4, the average $I_{\mathrm{ON}}$ for holes and electrons is shown. The $I_{\mathrm{ON}}$ increase linearly with the channel width, which is calculated as the perimeter of the SiNW, since volume inversion effect are negligible for SiNW-channel diameters larger than $10 \mathrm{~nm}$.

Conversely, Al-gated devices show a p-type conductance mainly (see Fig. 3(b)), which can be explained with Al contamination at pad regions due to the stencil process. This is also reflected in a $\mathrm{SS} \approx 200 \mathrm{mV} / \mathrm{dec}$, indicating that doping can effectively be used to improve SS by suppressing the ambipolar conductance. In both devices, $I_{\mathrm{ON}} / I_{\mathrm{OFF}}$ ratios of more than six orders of magnitude are observed. The lower current for the $n$-branch in the Al gated devices might be due to metal gate-induced doping. The transfer characteristic of pc-Si $\Omega$-gate inverters show (Fig. 5) an output voltage range $V_{\text {out }}$ compatible with the input voltage range and a gain of $\Delta V_{\text {out }} / \Delta V_{\text {in }} \approx 4$. Thus, pc-Si gated inverters are suitable elements for cascading additional devices, enabling the construction of more complex circuits. Similarly, a current bias scheme for FinFETs can be used to obtain pseudo-inverter operation, which consist in using a current bias scheme as depicted in Fig. 6 . For pseudo-inverter operation a triangular voltage transfer 
characteristic is obtained, with very low power consumption $(\leqslant 1.2 \mathrm{pW})$. Data from different dies on the wafer show stability of the $V_{\text {in }}$ value for which inversion occurs $\left(V_{\text {th }}\right)$ under different current bias (see Fig. 7(a)). Moreover, as it is shown in Fig. 7(b), the maximum of $V_{\text {out }}$ increases linearly with the current bias. Al gated inverters and pseudo-inverters are currently under investigation.

\section{Conclusions}

A fully CMOS compatible fabrication flow using low temperature a-Si LPCVD and stencil lithography has been developed and proved suitable for SiNW FinFETs having ambipolar conductance. Nanowires with up to $62 \mu \mathrm{m}$ length with two separate gates have been successfully fabricated, giving space for complex circuit design. Moreover, the stencil lithography has been demonstrated for the first time as a suitable technique for metal gate patterning on 3D nanowire structures. The excellent performance of individual FinFETs and preliminary data of inverter functionality pave the way for the fabrication of more complex Si nanowire FinFET circuits. Further characterization of Al stenciled inverters and fabrication on high aspect ratio structures is envisaged as natural continuation of this work.

\section{Acknowledgments}

The authors would like to thank the CMI staff at EPFL for help with the fabrication. This work was partially founded by SNSF grant 200021-122168, SNSF grant 200021-132539 and SNSF "Ambizione" grant PZ00P2_121923.

\section{References}

[1] H. Iwai, Microelectron. Eng. 86 (2009) 7-9.

[2] J.P. Colinge, Solid-State Electron. 48 (6) (2004) 897-905.

[3] M.R.T. Mofrad, J. Derakhshandeh, R. Ishihara, A. Baiano, J. van der Cingel, K. Beenakker, Jpn. J. Appl. Phys. 48 (3) (2009)

[4] R. Martel, V. Derycke, C. Lavoie, J. Appenzeller, K.K. Chan, J. Tersoff, et al., Phys. Rev. Lett. 87 (25) (2001).

[5] M.H. Ben-Jamaa, K. Mohanram, G.D. Micheli, Novel library of logic gates with ambipolar CNTFETs: opportunities for multi-level logic synthesis, in: 2009 Design, Automation and Test in Europe, 2009, pp. 622-627.

[6] V. Savu, J. Kivioja, J. Ahopelto, J. Brugger, IEEE Trans. Appl. Supercond. 19 (3) (2009).

[7] D. Sacchetto, M. Ben-Jamaa, G. DeMicheli, Y. Leblebici, Fabrication and characterization of vertically stacked gate-all-around Si nanowire FET arrays, in: Solid State Device Research Conference, ESSDERC '09, Proceedings of the European, 2009, pp. 245-248.

[8] G. Nakagawa, T. Asano, Jpn. J. Appl. Phys. 48 (6) (2009).

[9] V. Savu, S. Neuser, G. Villanueva, O. Vazquez-Mena, K. Sidler, J. Brugger, J. Vac. Sci. Technol. B: Microelectron. Nanomet. Struct. 28 (2010).

[10] D. Tsamados, N.V. Cvetkovic, K. Sidler, J. Bhandari, V. Savu, J. Brugger, et al., Solid-State Electron. 54 (9) (2010) 1003-1009. 